\title{
The Development of Female Sexual Behavior Requires Prepubertal Estradiol
}

\author{
Olivier Brock, ${ }^{1,3}$ Michael J. Baum, ${ }^{2}$ and Julie Bakker ${ }^{1,3,4}$ \\ ${ }^{1}$ Groupe Interdisciplinaire de Génoprotéomique Appliquée (GIGA)-Neurosciences, University of Liege, 4000 Liege, Belgium, ${ }^{2}$ Department of Biology, \\ Boston University, Boston, Massachusetts $02215,{ }^{3}$ Netherlands Institute for Neuroscience (NIN), 1105 BA Amsterdam, The Netherlands, and ${ }^{4}$ Medical \\ Psychology, Vrije Universiteit Medical Center, 1007 MB Amsterdam, The Netherlands
}

The classic view of brain and behavioral sexual differentiation holds that the neural mechanisms controlling sexual behavior in female rodents develop in the absence of ovarian sex hormone actions. However, in a previous study, female aromatase knock-out (ArK0) mice, which cannot convert testosterone to estradiol, showed deficient male-oriented partner preference and lordosis behaviors in response to adult ovarian hormones, raising the possibility that estradiol may contribute to the development of these female sexual behaviors. In the present experiments, administering estradiol prepubertally [between postnatal day 15 (P15) and P25] significantly enhanced the ability of ArKO female mice to display lordosis behavior in response to ovarian hormones administered later in adulthood, whereas treatment with estradiol over an earlier postnatal period (P5-P15) had no such effect. Treatment of ArK0 females with estradiol between P15 and P25 also rescued their later preference to approach distal cues from an intact male over an estrous female. ArKo females also displayed significantly less female-directed (male-typical) mounting behavior than wild-type control females when treated with testosterone in adulthood. Prepubertal estradiol treatment failed to reverse this deficit in ArKO females, whereas earlier postnatal estradiol augmented later mounting in both genotypes. Our results provide new evidence for an organizing role of prepubertal estradiol in the development of neural mechanisms that control female-typical sexual behavior.

\section{Introduction}

The classic view of brain sexual differentiation holds that the mammalian female nervous system develops in the absence of any active sex steroid signaling, whereas the male requires testosterone $(\mathrm{T})$, secreted over a species-specific perinatal period, to organize neural circuits that later control male-typical sexual behaviors. Furthermore, strong evidence suggests that many of the organizational actions of $\mathrm{T}$ result from estradiol $\left(\mathrm{E}_{2}\right)$ formed via neural aromatization of T (Baum et al., 1990; De Vries and Simerly, 2002). However, a contribution of perinatal $E_{2}$ to neural differentiation in female mammals was first proposed by ToranAllerand (1976), who found that $\mathrm{E}_{2}$ promoted neurite outgrowth from fetal hypothalamic explants of both sexes. Furthermore, several studies (Lisk, 1969; Gerall et al., 1973; Döhler et al., 1984; Baum and Tobet, 1986) suggested that perinatal $E_{2}$ could contribute to female brain and behavioral development. Eventually, however, the hypothesis languished due to the absence of conclusive supportive evidence. The creation of the aromatase knockout (ArKO) mouse, carrying a targeted mutation in the Cyp19

\footnotetext{
Received Jan. 13, 2011; revised Feb. 16, 2011; accepted Feb. 16, 2011.

Author contributions: 0. B. and J.B. performed research; 0. B. and J.B. analyzed data; $0 . B .$, M.J.B., and J.B. wrote the paper; M.J.B. and J.B. designed research.

This work was supported by a National Institutes of Health grant (HD044897) to M.J.B. and J.B., and by two grants from the Fonds National de la Recherche Scientifique (FNRS), Belgium (MIS F.4502.07, FRSM 3.4571.10) to J.B. J.B. is a Research Associate of the FNRS.

Correspondence should be addressed to Dr. Julie Bakker, Research Associate FNRS, GIGA-Neurosciences, University of Liege, Avenue de l'Hôpital 1 (B36), 4000 Liege, Belgium. E-mail: jbakker@ulg.ac.be.

DOI:10.1523/JNEUROSCI.0209-11.2011

Copyright $\odot 2011$ the authors $\quad 0270-6474 / 11 / 315574-05 \$ 15.00 / 0$
}

gene which renders animals of both sexes unable to convert T into $\mathrm{E}_{2}$ (Fisher et al., 1998; Honda et al., 1998), allowed us to reassess the role of $\mathrm{E}_{2}$ in the development of the female brain. Female ArKO mice, ovariectomized in adulthood and subsequently treated with $\mathrm{E}_{2}$ and progesterone $\left(\mathrm{P}_{4}\right)$, displayed less lordosis behavior than wild-type (WT) females (Bakker et al., 2002). Furthermore, ArKO females spent less time investigating volatile body odors from conspecifics of either sex (Bakker et al., 2002). These data suggest a potential role of $\mathrm{E}_{2}$ in female behavioral differentiation. We recently identified an estrogen-sensitive period during female development by using the expression of an $\mathrm{E}_{2}$-dependent neural gene, the progestin receptor (PR) (Brock et al., 2010). The amount of hypothalamic PR-immunoreactivity was significantly lower in female ArKO mice than in WT females at several prepubertal ages including postnatal day 15 (P15), P20, and $\mathrm{P} 25$, but not at earlier postnatal ages (P0, P5, or P10). Thus $\mathrm{E}_{2}$ may contribute to female-typical sexual differentiation perhaps beginning after P7 when the mouse ovary first produces $\mathrm{E}_{2}$ (Mannan and O'Shaughnessy, 1991).

We asked whether either early postnatal (P5-P15) (experiment 1) or prepubertal (P15-P25) (experiment 2) treatment with $\mathrm{E}_{2}$ would reverse later deficits in lordosis behavior and the investigation of potential sexual partners in ArKO females tested following adult treatment with $\mathrm{E}_{2}$ and $\mathrm{P}_{4}$. Female mice normally display surprisingly high levels of male-like mounting behavior toward other females around the time of estrus; they also show high levels of mounting after adult ovariectomy and T treatment (Edwards, 1971; Martel and Baum, 2009). We (Bakker et al., 2002) previously observed reduced mounting in ArKO females, 
suggesting that $\mathrm{E}_{2}$ normally masculinizes aspects of sexual behavior in female mice. Therefore we also asked whether early postnatal (experiment 1) or prepubertal (experiment 2) $E_{2}$ treatment would stimulate later mounting in ArKO females following adult treatment with $\mathrm{T}$.

\section{Materials and Methods}

Experimental animals

ArKO mice were generated by targeted disruption of exons 1 and 2 of the Cyp 19 gene (Honda et al., 1998). Heterozygous males and females of the CD1 strain were bred to generate WT and homozygous-null (ArKO) offspring at the GIGA-Neurosciences, University of Liège, Belgium. Food ("phytoestrogen-free" mouse chow D10001 AIN-76A, Brogaarden) and water were available to mice ad libitum. Pregnant females were checked daily for parturition ( $\mathrm{P} 0$ ) toward the end of pregnancy. All female subjects were weaned at P35 and genotyped by PCR analysis of tail DNA.

All male and female stimulus mice were derived from a local CD1 breeding colony. Stimulus females were ovariectomized in adulthood under general anesthesia and implanted with a 5-mm-long SILASTIC capsule (inner diameter, $1.57 \mathrm{~mm}$; outer diameter, $2.41 \mathrm{~mm}$ ) containing $17 \beta$-estradiol (for more details, see Bakker et al., 2002). Beginning several weeks later, subjects were brought into behavioral estrus on several occasions by added treatment with $\mathrm{P}_{4}(500 \mu \mathrm{g} /$ mouse $) 3 \mathrm{~h}$ before each test session.

All experiments were conducted in accordance with the guidelines set forth by the National Institutes of Health "Guide for the Care and Use of Laboratory Animals, Eighth Edition," and were approved by the Ethical Committee for Animal Use of the University of Liege.

\section{Early postnatal and prepubertal hormonal treatments}

A first cohort (experiment 1) of WT and ArKO females was injected subcutaneously over the early postnatal ages of P5-P15 with either estradiol benzoate (EB) $(0.5 \mu$ g every $2 \mathrm{~d}$ ) or sesame oil; additional groups of WT and ArKO females were not injected during this period. The latter group was included to control for any potential stress induced by repeated injections during early development. A second cohort (experiment 2) of WT and ArKO females was injected subcutaneously over prepubertal ages P15-P25 with either EB ( $0.05 \mu$ g every day) or sesame oil; additional females were not injected during this period. At 6 weeks of age, all female subjects in both cohorts were ovariectomized and implanted with an $\mathrm{E}_{2}$ capsule (details above). All behavioral testing began when subjects were 9 weeks of age. Since we never observed any statistically significant differences in aspects of behavior between the experimental groups in either cohort that were given oil vehicle injections versus no injections, we combined the data for the oil-treated and nontreated subjects into a single "control" group within the WT and ArKO females used for each experiment.

\section{Behavioral analyses}

All tests of behavior were performed beginning $6 \mathrm{~h}$ after the onset of the dark phase of the light/dark cycle (reversed $12 \mathrm{~h}$ light/dark cycle, lights off at 8:00 A.M.).

Lordosis behavior. Weekly lordosis tests were conducted in a Plexiglas aquarium ( $35 \mathrm{~cm}$ long $\times 25 \mathrm{~cm}$ high $\times 19 \mathrm{~cm}$ wide). A sexually experienced male was placed alone in the aquarium and allowed to adapt for 15 min. Subsequently, $3 \mathrm{~h}$ after receiving a $\mathrm{P}_{4}$ injection $(500 \mu \mathrm{g})$, the lordosis responses of the female to the mounts of the stimulus male were recorded. The test lasted until the female received 20 mounts or $15 \mathrm{~min}$ had elapsed. A lordosis quotient (LQ) was calculated by dividing the number of lordosis responses displayed by the female subjects by the number of mounts received $(\times 100)$.

Mate preference test. To assess mate preferences shown in response to auditory and/or olfactory stimuli, we used a box $(60 \times 13 \times 30 \mathrm{~cm})$ that was divided into three compartments using perforated opaque partitions. An intact male stimulus and an estrous female stimulus were placed in the lateral compartments with their own bedding to make the stimuli as odorous as possible. Three hours after receiving a $\mathrm{P}_{4}$ injection $(500 \mu \mathrm{g})$, the female subject was introduced into the middle compartment, and was observed for $10 \mathrm{~min}$. The time the subject spent poking her nose through the holes of the partition or actively sniffing the bottom of the partition in front of the female versus male stimulus animal was recorded.

Mounting behavior. After completing the above tests, we replaced the $\mathrm{E}_{2}$ capsule by one (same dimensions) containing $\mathrm{T}$. Two weeks later, tests were conducted in a Plexiglas aquarium. A female subject was placed alone in the aquarium and allowed to adapt for $15 \mathrm{~min}$. Subsequently, an estrous stimulus female was introduced and the number of mounts including pelvic thrusting movements shown by the female subject was scored for $30 \mathrm{~min}$.

\section{Experiment 1: EB treatment from P5 to P15}

Groups were first tested for lordosis behavior. A minimum of four tests is normally required to observe significant levels of lordosis behavior (LQ > 50) in WT females (Bakker et al., 2002; Kudwa and Rissman, 2003). However, we stopped after three lordosis tests since none of the females given early postnatal EB showed any lordosis behavior, and actually showed increased aggressive behavior toward the stimulus males, whereas WT controls already showed substantial levels of lordosis behavior (LQ > 50). We also did not assess mate preferences in this cohort of animals because no restoration in lordosis behavior was observed in EB-treated ArKO animals. Next, each group received a T implant and was tested once for mounting behavior with an estrous female.

\section{Experiment 2: EB treatment from P15 to P25}

Groups were first tested for lordosis behavior. Since we observed that lordosis behavior continued to increase in the fourth test in EB-treated ArKO females, we conducted a total of six lordosis tests over a 6-week period. Next, mate preferences were determined once following treatment with $\mathrm{E}_{2}$ and $\mathrm{P}_{4}$. Then, all subjects received a $\mathrm{T}$ implant and were subsequently tested once for mounting behavior 2 weeks later.

\section{Statistical analysis}

All behavioral data were analyzed using repeated measures ANOVA (Statistica 8.0 ) using genotype (2) and early postnatal or prepubertal treatment (2) as independent variables and tests as the repeated measure. When appropriate, all ANOVAs were followed by Fisher's least significant difference post hoc comparisons adapted for repeated-measures ANOVA. Only significant $(p<0.05)$ effects detected by the ANOVAs are presented here. To analyze mounting behaviors or results of single lordosis test sessions, we performed a two-way ANOVA [genotype (2) $\times$ treatment (2)].

\section{Results}

\section{Experiment 1: EB treatment from P5 to P15}

Lordosis behavior

As found by Bakker et al. (2002), ArKO control females showed significantly less lordosis than WT control females. This deficit was not rescued in ArKO females by early postnatal P5-P15 EB treatment. Indeed, treatment of WT females with EB over this period defeminized their lordosis behavior (Fig. 1A). ANOVA showed a significant interaction between genotype and treatment in test $3\left(F_{(1,34)}=5.34, p=0.03\right)$. Post hoc analyses indicated that lordosis quotients were lower in ArKO control females and in WT and ArKO females treated with EB compared with WT control females.

\section{Mounting behavior}

As found by Bakker et al. (2002), ArKO control females showed significantly less mounting than WT control females. EB treatment between P5 and P15 strongly increased mounting behavior in both ArKO and WT females following adult treatment with $\mathrm{T}$ (Fig. 2A). ANOVA showed a significant effect of treatment $\left(F_{(1,27)}=59.8, p<0.001\right)$ indicating that all females treated with EB during the early postnatal period displayed more mounts in adulthood. When we only compared control groups, ANOVA revealed a significant effect of genotype $\left(F_{(1,22)}=11.65, p=\right.$ 


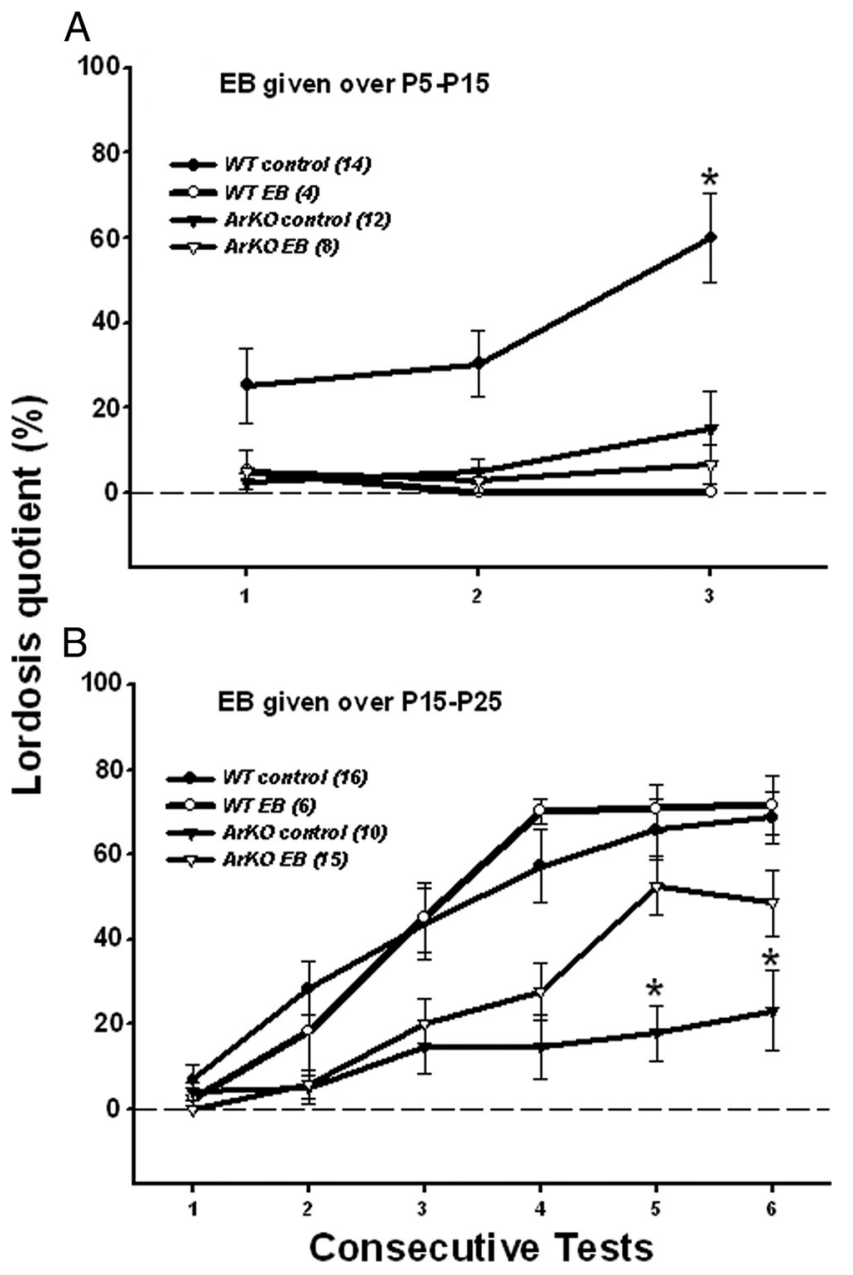

Figure 1. Effect of early postnatal $(\boldsymbol{A} ; \mathrm{P5}-\mathrm{P} 15)$ or prepubertal $(\boldsymbol{B} ; \mathrm{P} 15-\mathrm{P} 25)$ EB treatment of WT and ArKO female mice on the expression of lordosis behavior in adulthood following ovariectomy and treatment with ovarian hormones. Mean \pm SEM lordosis quotients over consecutive tests in WT control females, WT females treated with EB, ArKO control females, and ArKO females treated with $E B$ are shown. $A$, Values for WT control females on test 3 were significantly higher than values for the other 3 groups of female mice $\left({ }^{*} p<0.05\right)$ by post hoc tests. $\boldsymbol{B}$, Values for the ArKO control females on tests 5 and 6 were significantly lower than values for the other 3 groups of female mice $\left({ }^{*} p<0.05\right)$ by post hoc tests. The number of female mice in each group is given in parentheses.

0.002 ) indicating that WT control females showed more mounting behavior than ArKO control females.

\section{Experiment 2: EB treatment from P15 to P25}

Lordosis behavior

EB treatment from P15 to P25 almost completely rescued the later ability of ArKO females to display lordosis behavior, whereas EB given over this prepubertal period had no defeminizing effects on lordosis behavior in WT females (Figure $1 B$ ). Repeated-measures ANOVA on lordosis quotients over the six consecutive tests showed a significant interaction among test, genotype and treatment $\left(F_{(5,205)}=4.01, p=0.002\right)$. Post hoc analyses of data from tests 5 and 6 indicated that lordosis quotients were significantly lower in ArKO control females compared with the three other groups, whereas lordosis quotients were equivalent among EB-treated ArKO females, WT control females, and EB-treated WT females. Significant increments in lordosis quotients occurred, to differing degrees, with repeated testing in all 4 groups. Thus, post hoc analyses indicated that

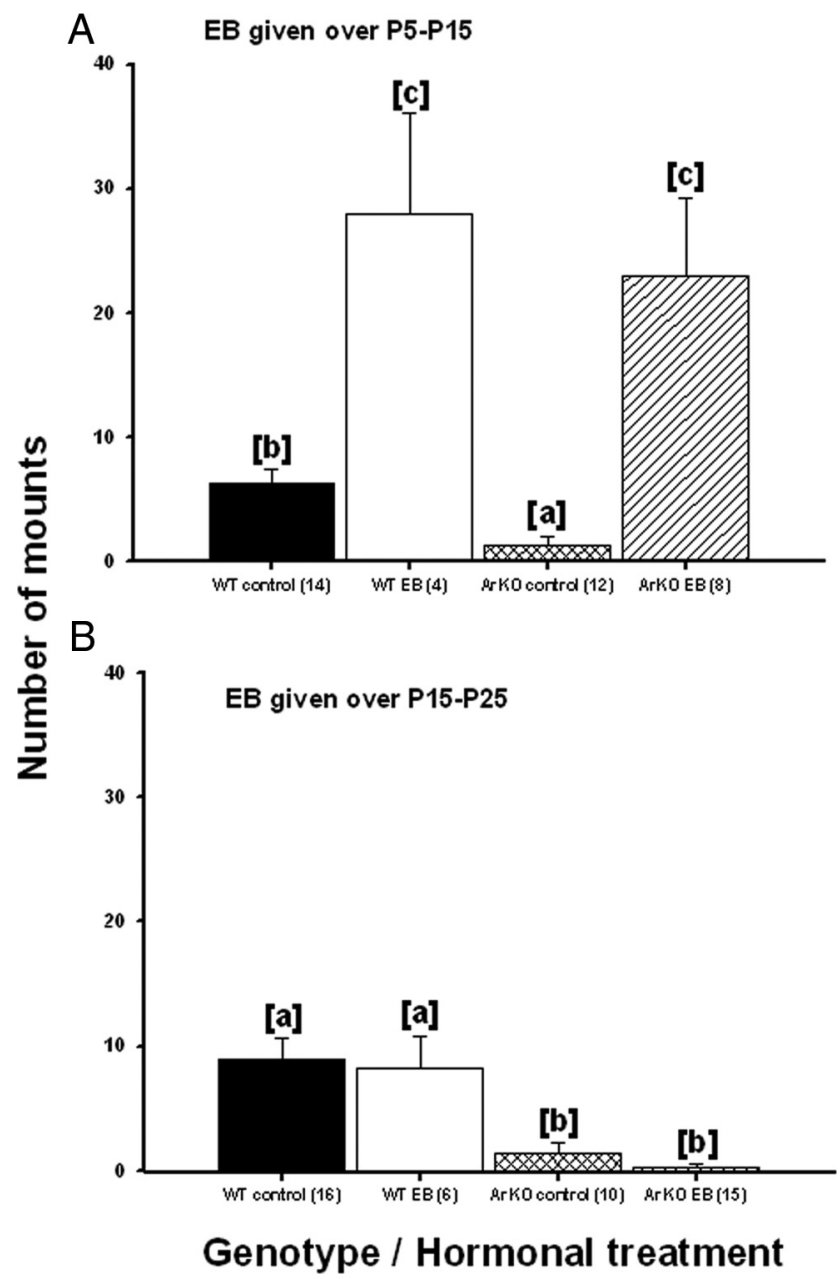

Figure 2. Effect of early postnatal $(\boldsymbol{A} ; \mathrm{P5}-\mathrm{P} 15)$ or prepubertal $(\boldsymbol{B} ; \mathrm{P} 15-\mathrm{P} 25)$ EB treatment of WT and ArKO female mice on the later display of female-directed mounting behavior in adulthood following ovariectomy and treatment with testosterone. Mean \pm SEM numbers of mounts displayed by WT control females, WT females treated with EB, ArKO control females, and ArKO females treated with EB are shown. Means with different superscript letters are significantly different from each other by post hoc comparisons $(p<0.05)$. The number of female mice in each group is given in parentheses.

lordosis quotients increased significantly from test 1 to test 4 before reaching plateau values in tests 5 and 6 in WT control and EB-treated WT females, whereas they increased significantly from test 3 to test 5 in EB-treated ArKO females and from test 1 to test 5 in ArKO controls.

\section{Mate preference test}

EB-treated ArKO female mice, like WT control and EB-treated WT females, showed a strong preference for an intact male over an estrous female, whereas ArKO control females showed no clear preference to approach male versus female stimulus animals (Fig. 3). This was confirmed by ANOVA on the time spent investigating the male versus female compartment. A significant interaction among stimulus, genotype and prepubertal treatment $\left(F_{(1,40)}=8.19, p=0.007\right)$ was obtained. Post hoc analyses indicated that WT control and EB-treated WT and ArKO females spent more time investigating the compartment containing the intact male versus the estrous female, whereas ArKO control females spent equal time investigating the two compartments.

\section{Mounting behavior}

Again, ArKO control females showed significantly less mounting behavior than WT control females following adult treatment 


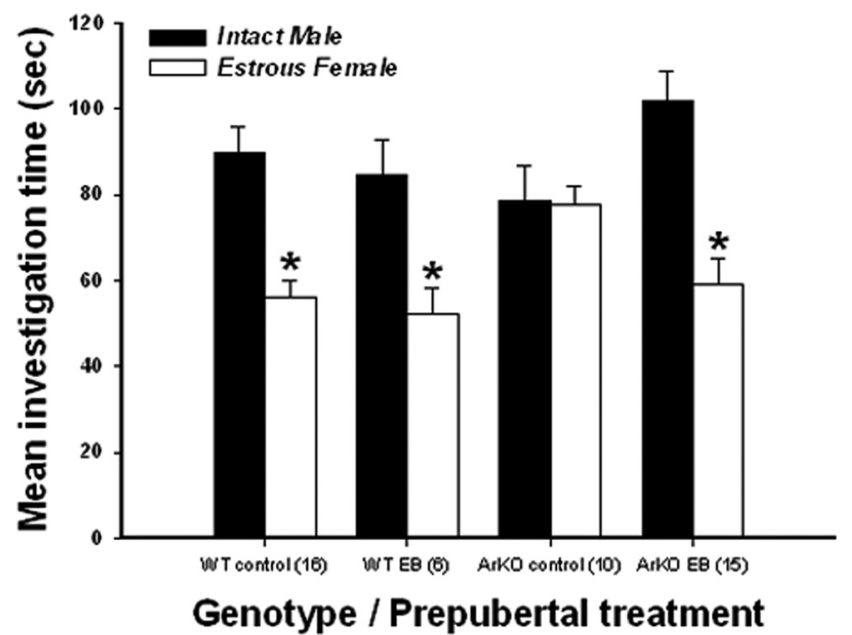

Figure 3. Effect of prepubertal (P15-P25) EB treatment of WT and ArKO females on mate preference in adulthood following ovariectomy and treatment with ovarian hormones. Shown are mean \pm SEM times spent by WT control females, WT females treated prepubertally with EB, ArKO control females, and ArKO females treated prepubertally with EB investigating the compartment containing an intact male versus an estrous female. ${ }^{*} p<0.05$, post hoc comparisons of time spent investigating the two types of stimulus animals. The number of female mice in each group is given in parentheses.

with T. This deficit was also seen in EB-treated ArKO females, showing that prepubertal EB treatment did not rescue this behavior. Prepubertal treatment with EB also did not affect later mounting behavior in WT females (Fig. $2 B$ ). ANOVA showed a significant genotype effect $\left(F_{(1,43)}=26.15, p<0.001\right)$ indicating that WT female groups showed higher levels of mounting when paired with an estrous female than ArKO female groups, regardless of the prepubertal hormonal treatment received.

\section{Discussion}

An important organizational role for $\mathrm{E}_{2}$ in male-typical differentiation of rodent sexual behavior is widely accepted and indeed, was confirmed in the present experiments. However, until now it has not been clear whether $\mathrm{E}_{2}$ contributes to the female-typical development of sexual behaviors. The present study provides new evidence for such an active role of prepubertal $E_{2}$ in femaletypical differentiation of brain mechanisms controlling both appetitive and consummatory aspects of female sexual behavior. Thus the ability of ArKO female mice to show WT-like levels of lordosis behavior in response to adult ovarian hormones was almost completely rescued by daily treatment with $\mathrm{EB}$ over a specific prepubertal period, P15-P25, whereas EB given over an earlier postnatal period (P5-P15) had no such effect in ArKO females and actually abolished lordosis behavior in WT females. In addition, prepubertal EB treatment restored the motivation of ArKO females to prefer to approach distal cues from a stimulus male as opposed to another female. These findings suggest that the classic view of a default organization of female typical neural and behavioral characteristics must be revised. Evidence from a previous retrospective interview study (Downey et al., 1989) of Turner syndrome $(45, \mathrm{XO})$ women in whom the ovaries failed to develop are also consistent with, though not definitive proof of, an active role of $E_{2}$ in the development of female sexual behavior. The Turner sample, who (29 of 31 subjects) received exogenous ovarian hormones as young adults, was significantly less likely than a control group of ovary intact $(46, \mathrm{XX})$ women with constitutional short stature to have ever had a boyfriend or to have engaged in sexual activity with a man. Even though the Turner syndrome and control subjects were matched for short stature, there were additional cognitive and/or physical attributes of Turner Syndrome women (in addition to the absence of ovarian $E_{2}$ ) that could have contributed to observed group differences in psychosexual function. Further studies are needed in women and in other mammalian species to test the generality of our conclusion that prepubertal $\mathrm{E}_{2}$ is normally required for the development of female sexual behavior.

The classic view of the sexual differentiation of brain and behavior in male rodents holds that perinatal T exerts strong defeminizing effects on the neural mechanisms mediating sexual behavior, with these effects of $\mathrm{T}$ being mediated by $\mathrm{E}_{2}$ derived from local aromatization of $\mathrm{T}$. The present results confirm the defeminizing effects of neonatal $\mathrm{E}_{2}$ on the brain since lordosis behavior was completely suppressed in WT females given EB between P5 and P15. They also corroborate an early study showing that neonatal $\mathrm{T}$ and $\mathrm{E}_{2}$ were equally effective in suppressing sexual receptivity in female mice (Edwards and Thompson, 1970), and are in line with many additional studies conducted in additional rodent species (for review, see Wallen and Baum, 2002).

The classic view of brain sexual differentiation also holds that female-typical brain and behavior develop in the absence of any active hormone signaling. This conclusion was primarily based on the finding that male rodents neonatally deprived of $\mathrm{T}$ or $\mathrm{E}_{2}$ showed lordosis behavior when primed with $\mathrm{E}_{2}$ and $\mathrm{P}_{4}$ in adulthood (for review, see Baum, 1979). Along with the seminal work of Toran-Allerand (1976), the possible importance of $E_{2}$ in female-typical differentiation of sexual behavior was suggested by early behavioral studies (Lisk, 1969; Gerall et al., 1973) showing that female rats ovariectomized on the day of birth had lower lordosis quotients following adult treatment with $\mathrm{E}_{2}$ and $\mathrm{P}_{4}$ than females that either kept their ovaries (Lisk, 1969) or were implanted with ovaries from birth until postnatal day 60 (Gerall et al., 1973). These various behavioral results thus suggested that exposure to $\mathrm{E}_{2}$ over a postnatal interval between birth and the age of puberty facilitated the later capacity to display female sexual behavior; however, they did not provide incontrovertible evidence that $\mathrm{E}_{2}$ normally contributes to the development of female sexual behavior in female mammals. For instance, the effects of neonatal ovariectomy on the potential to show lordosis behavior later in life were only transient (Gerall et al., 1973). However, our observation (Bakker et al., 2002) of reduced lordosis behavior and lack of a male-directed mate preference in female ArKO mice resurrected the question of whether $\mathrm{E}_{2}$ actively contributes to the development of the female brain. The present results identify a specific prepubertal period during which $\mathrm{E}_{2}$ exerts a feminizing action on both appetitive and consummatory aspects of female sexual behavior in mice. Finally, prepubertal (P15-P25) EB treatment had no detrimental effect on the expression of lordosis behavior by WT females, whereas earlier neonatal (P5-P15) EB treatment strongly defeminized WT females. These results suggest that the defeminization of sexual behavior capacity that normally occurs in male mice is completed by the actions of $\mathrm{E}_{2}$ before $\mathrm{P} 15$.

Our finding in both experiments that WT control females showed more mounting behavior (after T treatment) than ArKO control females provide an endocrine explanation for why female mice normally show male-typical mating behavior and argue against a previous proposal (Kimchi et al., 2007) that female mice will only display male-like mounting behavior when adult function of vomeronasal sensory neurons is disrupted. Our observation that early postnatal (P5-P15) EB treatment strongly masculinized both WT and ArKO females confirms the classic view that neonatal $\mathrm{E}_{2}$, formed via the neural aromatization of circulating $\mathrm{T}$, 
masculinizes aspects of brain and behavior in male mice. The neural substrate that controls mounting behavior in adulthood could normally be masculinized in fetal females by the local aromatization of $\mathrm{T}$ derived either from fetal males lying adjacent to the developing female fetuses (vom Saal and Bronson, 1980) or from the placenta which could also be a significant source of $\mathrm{T}$, as was observed in fetal rats (Gibori and Sridaran, 1981; Vreeburg et al., 1983; Baum et al., 1991). In contrast with lordosis capacity and male-oriented mate preferences, prepubertal (P15-P25) EB treatment did not rescue mounting capacity in ArKO females nor did it masculinize this behavior in WT females. These results suggest that by P15 the critical period for the development of the mounting behavior neural substrate is completed; they also provide further evidence that the neural substrate for female- and male-typical sexual behaviors in mice have different responses to $\mathrm{E}_{2}$ across the first 4 weeks of life (for review, see Baum, 1979). The neural substrate for female-typical sexual behavior is defeminized by early postnatal $\mathrm{E}_{2}$ actions (P5-P15) and actively feminized by the same sex hormone acting prepubertally over P15-P25. By contrast, $\mathrm{E}_{2}$ acts solely over the early postnatal period $(\mathrm{P} 0-\mathrm{P} 15)$ to masculinize the substrate for male sex behavior. After P15, this male behavior substrate is impervious to $\mathrm{E}_{2}$ manipulation until adulthood, when this hormone, perhaps acting with $\mathrm{T}$, activates this behavior. Indeed, the high level of mounting shown in EBtreated ArKO females (P5-P15) confirms the ability of adult T to activate this behavior, even in the absence of its conversion to $\mathrm{E}_{2}$. It should be noted that, in male mice, organization of mounting behavior capacity is mediated, at least in part, by an androgen receptor-mediated process (Raskin et al., 2009). To date, no data are available on the effects of knocking out the androgen receptor on the adult expression of mounting behavior by female mice so it is unknown whether different hormonal mechanisms underlie the organization of the neural circuitry regulating mounting behavior in the 2 sexes.

\section{References}

Bakker J, Honda S, Harada N, Balthazart J (2002) The aromatase knock-out mouse provides new evidence that estradiol is required during development in the female for the expression of sociosexual behaviors in adulthood. J Neurosci 22:9104-9112.

Baum MJ (1979) Differentiation of coital behavior in mammals: a comparative analysis. Neurosci Biobehav Rev 3:265-284.

Baum MJ, Tobet SA (1986) Effect of prenatal exposure to aromatase inhibitor, testosterone, or antiandrogen on the development of feminine sexual behavior in ferrets of both sexes. Physiol Behav 37:111-118.

Baum MJ, Carroll RS, Cherrv JA, Tobet SA (1990) Steroidal control of behavioural, neuroendocrine and brain sexual differentiation: studies in a carnivore, the ferret. J Neuroendocrinol 2:401-418.

Baum MJ, Woutersen PJ, Slob AK (1991) Sex difference in whole-body androgen content in rats on fetal days 18 and 19 without evidence that androgen passes from males to females. Biol Reprod 44:747-751.

Brock O, Douhard Q, Baum MJ, Bakker J (2010) Reduced prepubertal expression of progesterone receptor in the hypothalamus of female aromatase knockout mice. Endocrinology 151:1814-1821.
De Vries GJ, Simerly RB (2002) Anatomy, development, and function of sexually dimorphic neural circuits in the mammalian brain. In: Hormones, brain and behavior (Pfaff DW, Arnold AP, Etgen AM, Fahrbach SE, Rubin RT, eds), pp 137-191. Amsterdam: Academic.

Döhler KD, Hancke JL, Srivastava SS, Hofmann C, Shryne JE, Gorski RA (1984) Participation of estrogens in female sexual differentiation of the brain; neuroanatomical, neuroendocrine and behavioral evidence. Prog Brain Res 61:99-117.

Downey J, Ehrhardt AA, Gruen R, Bell JJ, Morishima A (1989) Psychopathology and social functioning in women with Turner syndrome. J Nerv Ment Dis 177:191-201.

Edwards DA (1971) Neonatal administration of androstenedione, testosterone or testosterone propionate: effects on ovulation, sexual receptivity and aggressive behavior in female mice. Physiol Behav 6:223-228.

Edwards DA, Thompson ML (1970) Neonatal androgenization and estrogenization and the hormonal induction of sexual receptivity in rats. Physiol Behav 5:1115-1119.

Fisher CR, Graves KH, Parlow AF, Simpson ER (1998) Characterization of mice deficient in aromatase (ArKO) because of targeted disruption of the cyp19 gene. Proc Natl Acad Sci U S A 95:6965-6970.

Gerall AA, Dunlap JL, Hendricks SE (1973) Effect of ovarian secretions on female behavioral potentiality in the rat. J Comp Physiol Psychol 82: $449-465$.

Gibori G, Sridaran R (1981) Sites of androgen and estradiol production in the second half of pregnancy in the rat. Biol Reprod 24:249-256.

Honda S, Harada N, Ito S, Takagi Y, Maeda S (1998) Disruption of sexual behavior in male aromatase-deficient mice lacking exons 1 and 2 of the cyp19 gene. Biochem Biophys Res Commun 252:445-449.

Kimchi T, Xu J, Dulac C (2007) A functional circuit underlying male sexual behaviour in the female mouse brain. Nature 448:1009-1014.

Kudwa AE, Rissman EF (2003) Double oestrogen receptor alpha and beta knockout mice reveal differences in neural oestrogen-mediated progestin receptor induction and female sexual behaviour. J Neuroendocrinol 15:978-983.

Lisk RD (1969) Progesterone: biphasic effects on the lordosis response in adult or neonatally gonadectomized rats. Neuroendocrinology 5 : $149-160$.

Mannan MA, O'Shaughnessy PJ (1991) Steroidogenesis during postnatal development in the mouse ovary. J Endocrinol 130:101-106.

Martel KL, Baum MJ (2009) Adult testosterone treatment but not surgical disruption of vomeronasal function augments male-typical sexual behavior in female mice. J Neurosci 29:7658-7666.

Raskin K, de Gendt K, Duittoz A, Liere P, Verhoeven G, Tronche F, MhaoutyKodja S (2009) Conditional inactivation of androgen receptor gene in the nervous system: effects on male behavioral and neuroendocrine responses. J Neurosci 29:4461-4470.

Toran-Allerand CD (1976) Sex steroids and the development of the newborn mouse hypothalamus and preoptic area in vitro: implications for sexual differentiation. Brain Res 106:407-412.

vom Saal FS, Bronson FH (1980) Sexual characteristics of adult female mice are correlated with their blood testosterone levels during prenatal development. Science 208:597-599.

Vreeburg JT, Groeneveld JO, Post PE, Ooms MP (1983) Concentrations of testosterone and androsterone in peripheral and umbilical venous plasma of fetal rats. J Reprod Fertil 68:171-175.

Wallen K, Baum MJ (2002) Masculinization and defeminization in altricial and precocial mammals: Comparative aspects of steroid hormone action In: Hormones, Brain and Behavior (Pfaff DW, Arnold AP, Etgen AM, Fahrbach SE, Rubin RT, eds), pp 385-423. New York: Academic. 